\title{
Editorial: Cell Stress, Metabolic Reprogramming, and Cancer
}

\author{
Sergio Giannattasio ${ }^{1 *}$, Mario G. Mirisola ${ }^{2}$ and Cristina Mazzoni ${ }^{3}$ \\ $1 /$ nstitute of Biomembranes, Bioenergetics and Molecular Biotechnologies, National Research Council of Italy, Bari, Italy, \\ ${ }^{2}$ Department of Surgical, Oncological and Oral Sciences, Section of Medical Oncology, University of Palermo, Palermo, Italy, \\ ${ }^{3}$ Department of Biology and Biotechnologies "C. Darwin", Pasteur Institute-Cenci Bolognetti Foundation, Sapienza University \\ of Rome, Rome, Italy
}

Keywords: cancer, mitochondria, hypoxia-inducible factor 1 alpha, ataxia-telangiectasia mutated, L-lactate, glutamine, antioxidant response, epithelial-to-mesenchymal transition

\section{Editorial on the Research Topic}

\section{Cell Stress, Metabolic Reprogramming, and Cancer}

The hallmarks of cancer comprise six biological capabilities acquired during the multistep development of human tumors: sustaining proliferative signaling, evading growth suppressors, resisting cell death, enabling replicative immortality, inducing angiogenesis, and activating invasion and metastasis (1). Mitochondria, beyond being the site of aerobic respiration, are at the crossroads of a variety of metabolic and signaling pathways resulting key regulatory organelles in cell life and death decision. Thus, it is no surprise that genomic, functional, and structural mitochondrial alterations have been associated with cancer and that mitochondria have become a pharmacological target in cancer therapy (2). Proliferating tumor cells show increased glycolysis and convert the majority of glucose to L-lactate, even in normoxic conditions. This is known as the Warburg effect. Actually, in many tumors, mitochondria are not defective in oxidative phosphorylation, and in the last decade, the molecular basis of Warburg effect has been reconsidered in the context of a set of concerted changes in energy metabolism and mitochondrial function that support tumorigenesis. This process, referred to as reprogramming of energy metabolism, is an emerging hallmark of cancer development $(3,4)$. This Research Topic presents one review, five mini-reviews, and an opinion article on the achievements and perspectives of studies on important aspects of cancer cell metabolic reprogramming whose mechanisms and regulation are still largely elusive. It also sheds light on certain novel functional components, which rewires cell metabolism in tumor transformation.

Metabolic reprogramming is driven by oncogenic changes of specific cell-signaling pathways and tumor microenvironment (5). The Mini-Reviews by Iommarini et al. (6) and Dahl and Aird (7) highlight what is currently known about the non-canonical function and regulation of hypoxia-inducible factor 1 alpha $(\mathrm{HIF}-1 \alpha)$ and ataxia-telangiectasia mutated (ATM) protein kinase, respectively. Iommarini et al. (6) review and discuss the non-canonical regulation of HIF$1 \alpha$ expression and stabilization in cancer cells, focusing on factors, which cause pseudohypoxia (HIF-1 $\alpha$ stabilization in normoxic conditions) or fail to stabilize HIF-1 $\alpha$ in low oxygen atmosphere (pseudonormoxia). The ATM protein kinase has been extensively studied for its role in the DNA damage response and its association with the disease ataxia telangiectasia. Dahl and Aird's review (7) highlights our current knowledge about ATM's regulation of carbon metabolism, the implication of these pathways in cancer, and the development of ATM inhibitors as therapeutic strategies for cancer.

It is well established that glucose is uniquely capable of supporting Warburg metabolism (or aerobic glycolysis), in which pyruvate is converted to lactate through a process that is coupled to ATP production in the cytoplasm. Such metabolic reprogramming and nutrient sensing is an elaborate way by which cancer cells respond to high bioenergetic and anabolic demands during tumorigenesis. 
Ždralević et al. (8) in their Mini-Review discuss the benefits and limitations of disrupting fermentative glycolysis at different levels of the pathway in order to find the most effective mode to overcome cancer cell metabolic plasticity that seriously limits the use of glycolysis inhibition for impeding tumor growth. With this respect, in view of the existence of a mitochondrial L-lactate dehydrogenase (m-L-LDH), Passarella and Shurr (9) propose in their Opinion a revision of the Cori cycle in all types of cells where mitochondrial metabolism of $\mathrm{L}$-lactate is active.

Beyond the shift of glucose metabolism to aerobic glycolysis, some cancer cells are considered "glutamine addicted" because their growth and proliferation rates depend on the availability of this amino acid. This, together with the role of amino acid metabolism in tumorigenesis, is one of the key aspects of cancer cell metabolism, which is still matter of intense investigations. The Review by Vučetić et al. (10) provides the first unified review on the amino acid dependency of cancer antioxidant defense, a topic that has received more attention recently. Furthermore, the MiniReview by Scalise et al. (11) provides a deep insight into glutamine transport and mitochondrial metabolism in cancer cell growth, highlighting glutamine transporters of plasma membrane, the key enzyme glutaminase, and other proteins involved glutamine metabolism as novel targets for anti-cancer drug development.

Beyond the metabolic shift toward glycolysis, typical of cancer cells, several evidences have shown that mitochondrial dysfunction provides survival advantage to cancer cells, suggesting that mitochondria have a tumor suppressor function (5). Mitochondrial dysfunction has been implicated in cancer chemoresistance (12). The association between mitochondrial dysfunction and progression to a metastatic phenotype is gradually emerging. Epithelial-to-mesenchymal transition (EMT) allows epithelial cancer cells to assume mesenchymal features, endowing

\section{REFERENCES}

1. Hanahan D, Weinberg RA. Hallmarks of cancer: the next generation. Cell (2012) 144:646-74. doi:10.1016/j.cell.2011.02.013

2. Picard M, Wallace DC, Burelle Y. The rise of mitochondria in medicine. Mitochondrion (2016) 30:105-16. doi:10.1016/j.mito.2016.07.003

3. Pavlova NN, Thompson CB. The emerging hallmarks of cancer metabolism. Cell Metab (2016) 23:27-47. doi:10.1016/j.cmet.2015.12.006

4. Vander Heiden MG, DeBerardinis RJ. Understanding the intersections between metabolism and cancer biology. Cell (2017) 168:657-69. doi:10.1016/j. cell.2016.12.039

5. Vyas S, Zaganjor E, Haigis MC. Mitochondria and cancer. Cell (2016) 166:555-66. doi:10.1016/j.cell.2016.07.002

6. Iommarini L, Porcelli AM, Gasparre G, Kurelac I. Non-canonical mechanisms regulating hypoxia-inducible factor 1 alpha in cancer. Front Oncol (2017) 7:286. doi:10.3389/fonc.2017.00286

7. Dahl ES, Aird KM. Ataxia-Telangiectasia mutated modulation of carbon metabolism in cancer. Front Oncol (2017) 7:291. doi:10.3389/fonc.2017.00291

8. Ždralević M, Marchiq I, Cunha de Padua MM, Parks SK, Pouysségur J. Metabolic plasiticy in cancers-distinct role of glycolytic enzymes GPI, LDHs or membrane transporters MCTs. Front Oncol (2017) 7:313. doi:10.3389/fonc.2017.00313

9. Passarella S, Schurr A. 1-Lactate transport and metabolism in mitochondria of Hep G2 cells-The Cori cycle revisited. Front Oncol (2018) 8:120. doi: $10.3389 /$ fonc. 2018.00120

10. Vučetić M, Cormerais Y, Parks SK, Pouysségur J. The central role of amino acids in cancer redox homeostasis: vulnerability points of the cancer redox code. Front Oncol (2017) 7:319. doi:10.3389/fonc.2017.00319 them with enhanced motility and invasiveness, thus enabling cancer dissemination and metastatic spread. The Mini-Review by Guerra et al. (13) in this Research Topic gives an overview on the mechanistic link between EMT and mitochondrial dysfunction fostering the identification of the molecular determinants of the mitochondria-nucleus communication network linking mitochondrial dysfunction with EMT activation, which may provide useful therapeutic targets for treatment and prevention of metastatic cancer.

The contributions to this Research Topicdeal with investigations at the leading edge of cancer research and provide an overview on key cellular processes and components, which are the basis of metabolic reprogramming of cancer cells. Inflammation has also been recognized as a hallmark of cancer and is known to play an essential role in the development and progression of most cancers, even those without obvious signs of inflammation and infection (14). Warburg metabolism is a hallmark of immune cells that have the potential to cause inflammation. Recently, Kornberg et al. gave proof of concept that aerobic glycolysis is a therapeutic target for regulating inflammation (15), further confirming the possibility that targeting key enzymes within metabolic pathways will provide new therapeutic options for cancer.

This Research Topic brings witness that research on metabolic reprogramming of cancer cells is coming of age and will still bring with it exciting results to lay the bases for the development of new therapies and the implementation of nutritional regimen for a healthy life as well as the improvement of anti-cancer therapies.

\section{AUTHOR CONTRIBUTIONS}

SG wrote the first draft of the manuscript; SG, MGM and CM contributed to manuscript revision.

11. Scalise M, Pochini L, Galluccio M, Console L, Indiveri C. Glutamine transport and mitochondrial metabolism in cancer cell growth. Front Oncol (2017) 7:306. doi:10.3389/fonc.2017.00306

12. Guerra F, Arbini AA, Moro L. Mitochondria and cancer chemoresistance. Biochim Biophys Acta (2017) 1858:686-99. doi:10.1016/j.bbabio.2017. 01.012

13. Guerra F, Guaragnella N, Arbini AA, Bucci C, Giannattasio S, Moro L. Mitochondrial dysfunction: a novel potential driver of epithelial-to-mesenchymal transition in cancer. Front Oncol (2017) 7:295. doi:10.3389/ fonc. 2017.00295

14. Taniguchi K, Karin M. NF-kappaB, inflammation, immunity and cancer: coming of age. Nat Rev Immunol (2018) 18:309-24. doi:10.1038/ nri.2017.142

15. Kornberg MD, Bhargava P, Kim PM, Putluri V, Snowman AM, Putluri N, et al. Dimethyl fumarate targets GAPDH and aerobic glycolysis to modulate immunity. Science (2018) 360:449-53. doi:10.1126/science.aan4665

Conflict of Interest Statement: The authors declare that the research was conducted in the absence of any commercial or financial relationships that could be construed as a potential conflict of interest.

Copyright (c) 2018 Giannattasio, Mirisola and Mazzoni. This is an open-access article distributed under the terms of the Creative Commons Attribution License (CC BY). The use, distribution or reproduction in other forums is permitted, provided the original author(s) and the copyright owner are credited and that the original publication in this journal is cited, in accordance with accepted academic practice. No use, distribution or reproduction is permitted which does not comply with these terms. 